\title{
Organic Mathematics: Then and Now
}

\section{Jonathan M. Borwein and Veselin Jungić}

In the early 1990s a group of researchers, J. Borwein, P. Borwein, R. Corless, L. Jörgenson, and N. Sinclair, all then affiliated with the Center for Experimental and Constructive Mathematics at Simon Fraser University-as part of a National Telelearning Network-started the Organic Mathematics Project (OMP). ${ }^{1}$ One of the main goals of the project was to achieve a more meaningful integration of the technologies then available, moving towards the ideal environment described as follows:

\begin{abstract}
A mathematician working in ideal conditions would be able to look at a fresh problem and easily access any related material, find all the work on simpler but similar problems, and quickly carry out any subcomputations needed for the solution of the fresh problem. Such a person would also be able to consult freely not only with colleagues but with experts with whom he or she/was not previously familiar.
\end{abstract}

The project culminated with the Organic Mathematics Workshop, held in Vancouver, B.C., on December 12-14, 1995. The editors of the online workshop proceedings ${ }^{2}$ stated that they

Jonathan M. Borwein is laureate professor and director at the Centre for Computer Assisted Research Mathematics and Its Applications (CARMA) at the University of Newcastle and distinguished professor at King Abdulaziz University. His email address is jonathan.borwein@ newcast le.edu. au.

Veselin Jungić is deputy director at the IRMACS Centre, Simon Fraser University. His email address is vjungic@ irmacs.sfu.ca.

${ }^{1}$ See http://www. cecm.sfu.ca/organics/contents. htm T.

${ }^{2}$ Also published in the traditional hard-copy form: Organic Mathematics (Burnaby, BC, 1995), CMS Conf. Proc. 20, Amer. Math. Soc., Providence, RI, 1997.

DOI: http://dx.doi.org/10.1090/noti805

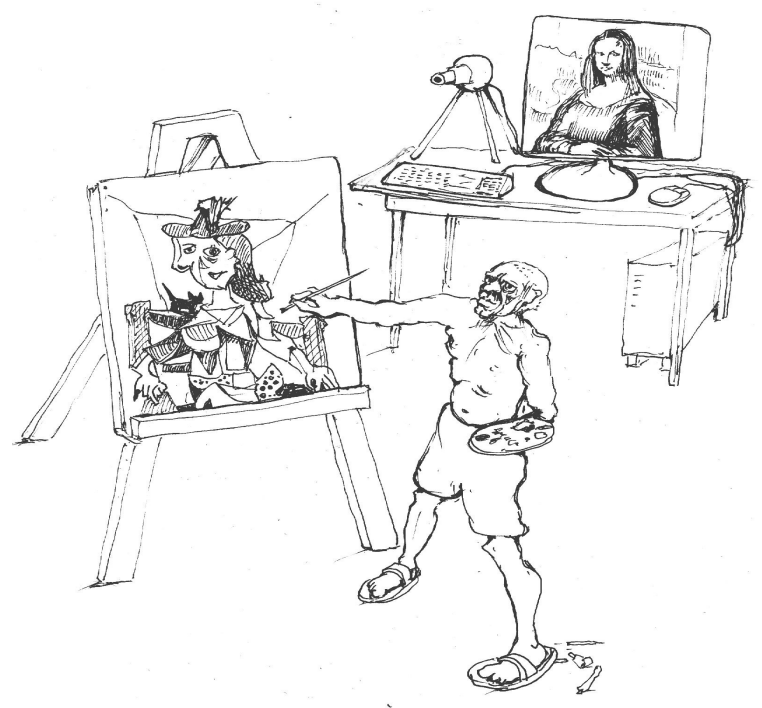

"Computers are useless. They can only give you answers."

want the information in the Proceedings of this workshop to form examples of "living documents", connected to their references, connected to each other, connected to algorithms for live mathematical work on the part of the reader. We want them to be, in a word, "organic".

The body of the OMP was the "mathactivations" ${ }^{3}$ of fifteen previously published and highly regarded papers by leading researchers such as George Andrews, Jeff Lagarias, Andrew Odlyzko, Ron Graham, and Andrew Granville. ${ }^{4}$ This allowed

${ }_{3}^{3}$ Algorithms in Maple, graphics, audio, videos, simula-
tions, annotation tools, etc. While we already used Java
in our Centre, we did not exploit it as it was not then well
established.
${ }^{4}$ See http://www. cecm.sfu.ca/organics/papers/for for
the papers, some of which had already won major prizes. 
the project to focus on issues of enhancement rather than traditional editing. There were an equal number of submitted papers which were similarly enhanced-in each case by teams of two students who worked with the author. ${ }^{5}$ In the section "The future of these proceedings" some of the challenges and dilemmas were listed.

Where will this volume be in ten years? How about ten months? Experience to date tells us that the potential for long-term functionality is quite limited. ... Perhaps a more pertinent question is, supposing that someone will be able to read these proceedings at some time in the future, will they want to ${ }^{6}$

Given that we were largely building a bridge as we walked upon it-since very few of today's Web design or management tools existed-it is fair to say that we have been pleased and surprised by the robustness and longevity of the OMP. Software licensing issues and antique servers have proven the main exceptions.

As one of many possible illustrations that some of the core ideas motivating the Organic Mathematics Project still intrigue mathematicians, we mention the Manifold Atlas Project ${ }^{7}$ sponsored by the Hausdorff Center at the University of Bonn. The structure of the Project is described in the following "organic" way:

The pages of the Atlas provide a public work space for topologists and other interested scientists to collaborate via the World Wide Web. Atlas pages are continually open for editing and development. However, they are not strongly scientifically citable. Once an Atlas page has reached maturity, it will be refereed. ... Atlas pages which have been published in the Bulletin are still open for improvement, modification, and correction. When such a page again reaches maturity, it will be refereed again.

Contemporary researchers and publishers who aim for "a more meaningful use of the technologies available" face challenges depressingly similar to those of nearly twenty years ago. It is an interesting exercise to compare two documents, one ${ }^{8}$

\footnotetext{
$\overline{5}$ And got course credit for so doing.

${ }^{6}$ We suggest the curious reader visit http://www. cecm. sfu.ca/organics/for answers to these questions.

'http://www.map.him.uni-bonn.de/Main_Page.

${ }^{8}$ A. M. Odlyzko, Tragic loss or good riddance? The impending demise of traditional scholarly journals, Journal of Universal Computer Science 0, 3-53.
}

published in 1994 and the other ${ }^{9}$ published in 2011 , on the self-same topic of the future of the mathematics journals.

For a modern working mathematician it is increasingly difficult to follow developments in his or her field. The quantity of published papers or preprints, the number of relevant meetings, the steady stream of information coming from sources such as the arXiv or blogs, ${ }^{10}$ and other Web-based sources, ${ }^{11}$ the ever changing working environment, increasing demand in teaching and administrative duties, sharp competition to publish new results, ${ }^{12}$ the exhausting process of applying for and reporting on research funds, and the search for high quality graduate students, can be nearly overwhelming for many members of our community.

Hence, for years to come, just to stay well informed "our hero" will be on the run like Alice's Red Queen ${ }^{13}$ to access needed information, to grasp it at the necessary level as quickly as possible, and to trust the sources of that information. We believe that mathematical journals, in their capacity as verifiers, evaluators, disseminators, and keepers of mathematical knowledge, should play a major role in helping the community to face some of the challenges listed above. This will include resolving some of the issues that are consequences of the new role that technology plays in mathematical research and of the ways that mathematics is and will be communicated.

Current technology has forever changed the relationship between so-called pure and applied mathematics. Using mathematical technology to predict or check a mathematical fact, to visualize abstract objects and/or their properties, or to calculate with unprecedented precision, has become a standard in mathematical research. The computational sides of graph theory, topology or group theory, applications of number theory, experimental mathematics, and mathematical modeling of complex systems are just a few of

\footnotetext{
${ }^{9}$ Mathematics journals: What is valued and what may change. Report of the workshop held at MSRI, Berkeley, California, on February 14-16, 2011, http:// www.msri.org/attachments/workshops/587/ MSRIfina1report.pdf.

${ }^{10}$ From sublime, such as Tim Gower's Polymath Project http://michae1nie1sen.org/polymath1/index. php?tit7e=Main_Page, to ridiculous.
${ }^{11}$ See, for example, http: //www. eg-models.de/,
${ }^{12}$ And even to prematurely announce them in the press.
13 "Well, in our country," said Alice, still panting a little, "you'd generally get to somewhere else if you run very fast for a long time, as we've been doing." "A slow sort of country!" said the Queen. "Now, here, you see, it takes all the running you can do, to keep in the same place. If you want to get somewhere else, you must run at least twice as fast as that!" From Lewis Carroll's Alice Through the Looking Glass.




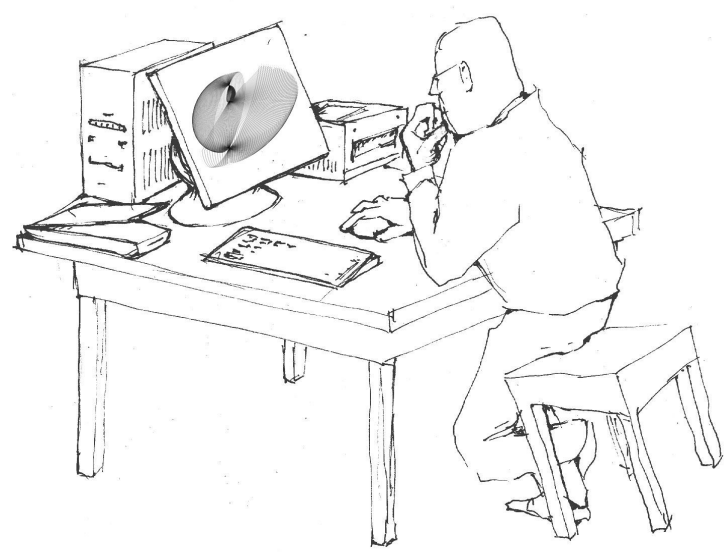

“The computer knows more than I do?”

the branches of mathematics that both depend heavily on the technology and inspire and demand its new developments.

Now contemporary natural and social scientists wish-rightly or wrongly - to quantify almost everything and need to recognize and record changes in their data. This gives a new responsibility to the mathematics community. This responsibility includes providing tools to analyze and understand collected data and to be able to correct theoretical errors, fill holes, and explain apparent paradoxes in the data. It follows that both mathematics driven by preexisting data and mathematics that produces new data are now part of everyday life in MathLand, and as such they deserve fair treatment in mathematics journals.

For example, we need to establish protocols as to how journals manage verifying, presenting, and storing various computational-or computationally checkable-components of newly created mathematics. As part of such protocols, standard suites of problems should be maintained for the validation of performance claims for new algorithms. ${ }^{14}$ It should no longer be good enough for an author to assert that his or her method is "better" or "competitive with" current standard methods. It should also be possible for a referee intrigued by or suspicious of a formula to try it on the spot.

Also, maintaining the data that support mathematical discovery is an issue that is both technical and part of the emerging new relationship between publishers, editors, and authors. How can we preserve and keep data available to interested readers, an issue complicated by changing protocols and related technology? Whose responsibility should this be, publishers' or authors'? In these matters we can learn much from the biomedical sciences.

\footnotetext{
${ }^{14}$ In many applied areas there are good suites of test problems. What is missing then is a protocol and infrastructure for their use.
}

Given all this, we should like to further revisit the notion of the ideal environment as it was envisioned by the Organic Mathematics Project in $1995 . .^{15}$ One of the reasons that we do this is because even now a paper published in a mathematical journal often looks worse than its arXiv version-cramped, unenhanced, and unloved. Moreover, the arXiv paper is often less fun and less informative than a good Beamer or PowerPoint presentation on the subject which has a human element-side comments, reflections, jokes-even when it lacks animations, simulations, movie clips, or the like, all of which have been possible for at least twenty years.

Now, it takes effort to add such enhancements, and while many of us already do so in part, it is unreasonable to expect this as a basic requirement for a mathematics paper. While the jobs of copyediting and typesetting have been added to the rest

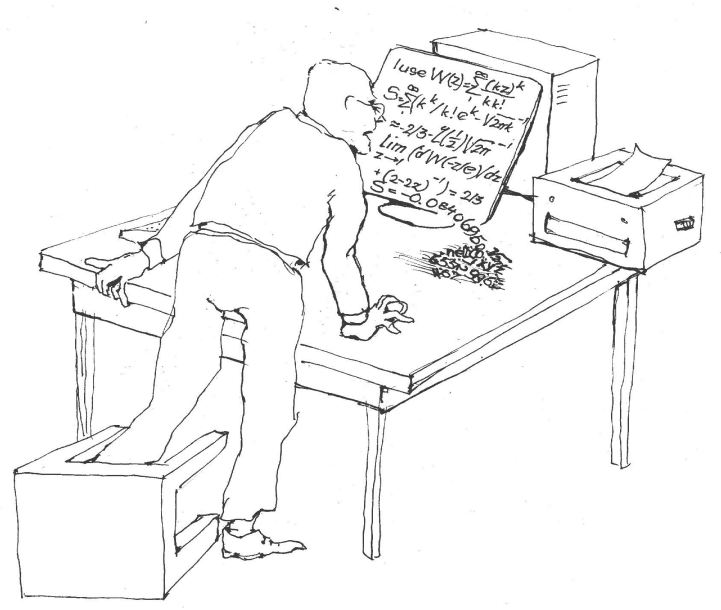

“By golly—she does!”

of the academic remit, the journals (even those of nice guys like SIAM and the AMS) have responded by offering us even less for more.

So here is what we suggest: SIAM, the AMS, and other society publishers should build us a modern publishing template-a "Beamer without tears" if you will-and promise to expedite publication of well-enhanced articles.

We believe that this kind of change will greatly benefit mathematicians and the scientific community in general. By creating mathematical publications in the form of layered structures, the reader will get an opportunity to match his or her needs and expertise with the appropriate layer of the publication. This would make new mathematics more accessible to many researchers from other parts of mathematics and other scientific fields and will help remove the barrier established by the

\footnotetext{
${ }^{15}$ What follows is partly based on a July 2011 letter in SIAM Review by the first author.
} 
fact that most mathematical papers are written by experts for experts.

In its essence this idea is not new. In 1994 Leslie Lamport wrote:

When first shown a detailed, structured proof, most mathematicians react: I don't want to read all those details; I want to read only the general outline and perhaps some of the more interesting parts. My response is that this is precisely why they want to read a hierarchically structured proof. The highlevel structure provides the general outline; readers can look at as much or as little of the lower-level detail as they want. ${ }^{16}$

The main difference between then and now is in the technology. For Lamport "[t]he ideal tool for reading a structured proof would be a computerbased hypertext system." Today, ways to separate different levels of a proof are near limitless.

We are aware that there is another, equally "organic", publishers' side of this issue. Let us list some of the questions that are central to the enter-
Who is to make these decisions: professional mathematicians, research librarians, academic publishers, governments through their granting agencies, or maybe its Majesty, the Market itself? Furthermore, would a mathematician, whose whole world had just crashed because he found a seemingly devastating bug in his proof, care about any of this?

The necessary components such as MathJax are all here if only recently. Yes, there are many annoying technical and organizational details, but all that is needed to overcome them is for academic publishers to step up to the plate for their own benefit and for the benefit of the whole mathematical and scientific community.

Acknowledgments. The final cartoon by Simon Roy shows recent computer-discovered work on random walks by the first author and his colleagues. All others are by Karl H. Hofmann and appeared originally in J. Borwein and K. Devlin, The Computer as Crucible, A K Peters, 2008.

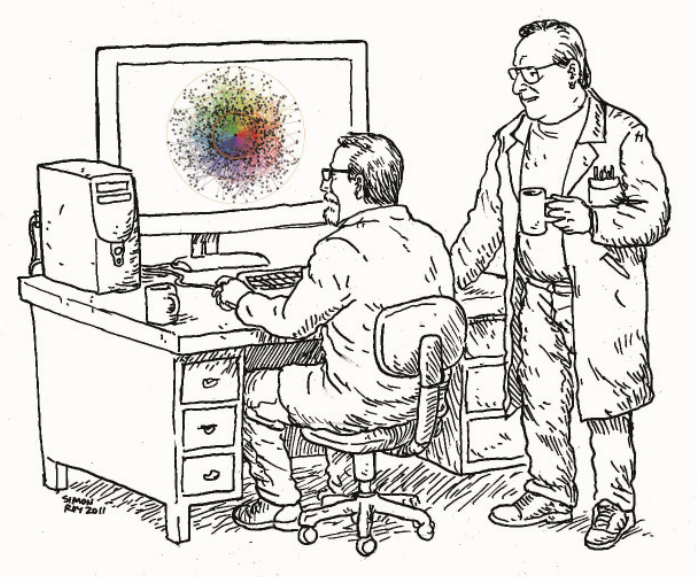

"Sometimes it is easier to see than to say."

prise of scientific publishing. How would the above proposed changes impact the existing business model for mathematics journals? Would further breaking with traditional modes of publishing be manageable for commercial publishers? Would the model "fit more than a few"? Would it be robust enough to keep the current level of mathematical rigor intact? Would it truly reach out of the experts' zone and, at the same time, justify the necessary investment in people and technology? Should the status quo be disturbed? Our answer to all of these questions is "yes".

\footnotetext{
${ }^{16}$ L. Lamport, How to write a proof, American Mathematical Monthly 102 (7) (1994), 600-608.
}

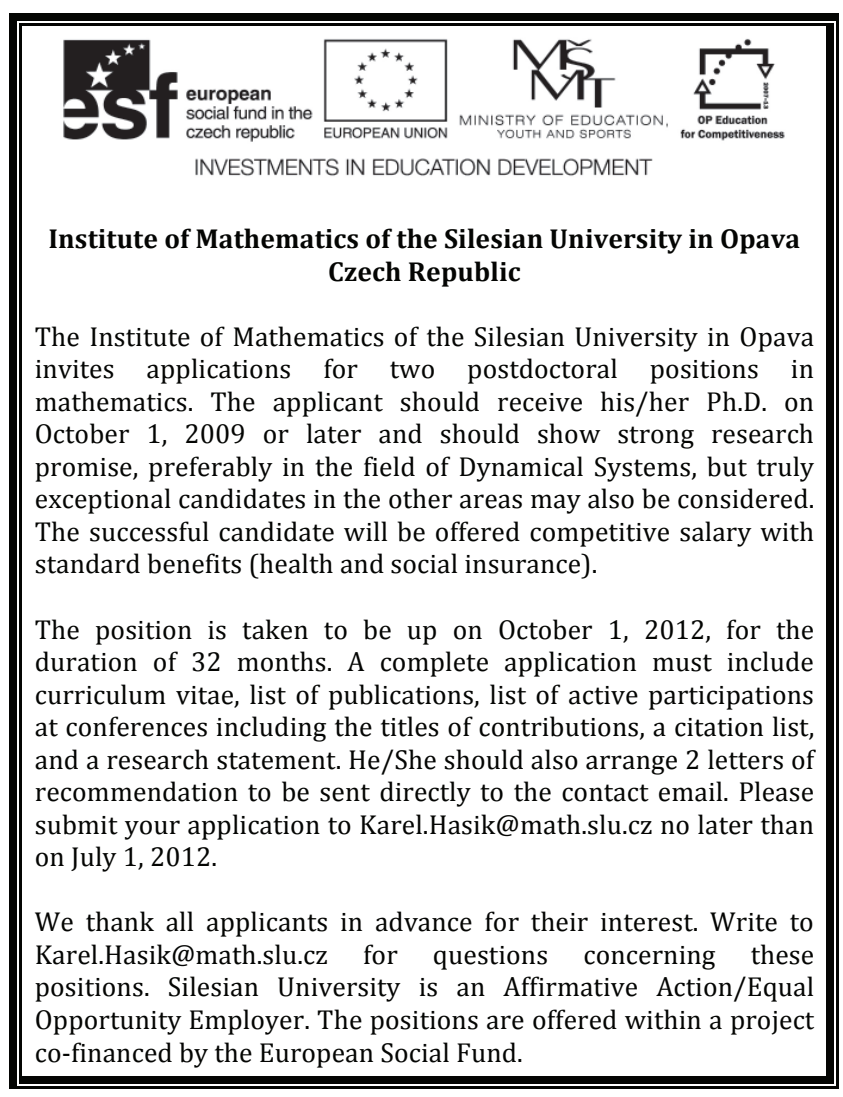

NOTICES OF THE AMS 